\title{
Out-of-hospital Cardiac Arrest: A Systematic Review of Current Risk Scores to Predict Survival
}

Ying X. GUE MBChB, MRCP, PhD ${ }^{1,2}$, Krishma ADATIA BSc MBChB ${ }^{2}$, Rahim KANJI

BSc MBBS, MRCP ${ }^{2,3}$, Tatjana POTPARA MD PhD ${ }^{4}$, Gregory YH LIP MD FRCP DFM ${ }^{5 *}$, Diana A. GOROG MD, PhD FRCP FESC ${ }^{1,2,3 *}$

*Joint senior authors

1. University of Hertfordshire, Hertfordshire, United Kingdom

2. Cardiology Department, East and North Hertfordshire NHS Trust, Stevenage, United Kingdom

3. National Heart and Lung Institute, Imperial College, London, United Kingdom

4. Clinical Centre of Serbia \& School of Medicine, Belgrade University, Serbia, Belgrade

5. Liverpool Centre for Cardiovascular Science, University of Liverpool and Liverpool Heart \& Chest Hospital, Liverpool, United Kingdom

Word count: 3145

Correspondence to:

Prof. Diana A Gorog

National Heart \& Lung Institute

Imperial College

Dovehouse Street

London SW3 6LY

United Kingdom

Email: d.gorog@imperial.ac.uk 


\section{Abbreviations}

ACLS - advanced cardiac life support

AUC - area under the receiver operating curve

$\mathrm{CPR}$ - cardiopulmonary resuscitation

GCS - Glasgow Coma Scale

ICU - Intensive care unit

NPV - Negative predictive value

OHCA - Out-of-hospital cardiac arrest

ROSC - Return of spontaneous circulation

PPV - Positive predictive value

TTM - therapeutic temperature management 


\begin{abstract}
Importance: The arrest and the post-arrest period are an incredibly emotionally traumatic time for family and friends of the affected individual. There is a need to assess prognosis early in the patient pathway to offer objective, realistic and non-emotive information to the next-of-kin regarding the likelihood of survival.
\end{abstract}

Objective: To present a systematic review of the clinical risk scores available to assess patients on admission following out-of-hospital cardiac arrest (OHCA) which can predict in-hospital mortality.

Evidence review: A systematic search of online databases Embase, MEDLINE and Cochrane Central Register of Controlled Trials was conducted up until 20th November 2020. Findings: Out of 1,817 initial articles, we identified a total of 28 scoring systems, with 11 of the scores predicting mortality following OHCA included in this review. The majority of the scores included arrest characteristics (initial rhythm and time to return of spontaneous circulation) as prognostic indicators. Out of these, the three most clinically-useful scores, namely those which are easy-to-use, comprise of commonly available parameters and measurements, and which have high predictive value are the OHCA, NULL-PLEASE and rCAST scores, which appear to perform similarly. Of these, the NULL-PLEASE score is the easiest to calculate and has also been externally validated.

Conclusion: Clinicians should be aware of these risk scores, which can be used to provide objective, non-emotive and reproducible information to the next-of-kin on the likely prognosis following OHCA. However, in isolation, these scores should not form the basis for clinical decision-making.

Word count: 242 words 


\section{Highlights}

- There is a need to assess prognosis early in the patient pathway to offer objective, realistic and non-emotive information to the next-of-kin regarding the likelihood of survival.

- There are a multitude of prognostic scores available in the literature.

- It is essential that clinicians are aware of useful scores, which can help objectively assess likely outcome and guide the information given to the next-of-kin.

- However, risk scores, in isolation, should not form the basis for clinical decision making. 


\section{Introduction}

Ischaemic heart disease remains the leading cause of death in developed countries and the predominant cause of cardiac arrest. In the United States, the incidence of emergency medical services-treated out-of-hospital cardiac arrest (OHCA) is approximately 74.3 per 100,000 population.(1) In 2018, of those individuals who experienced OHCA and had laypersoninitiated cardiopulmonary resuscitation (CPR), only 10.4\% survived to hospital discharge(1) and although survival to discharge is uniformly poor worldwide, regional disparities exist, ranging from $1-25 \% .(2,3)$ Not only is OHCA a leading cause of mortality but also morbidity, accounting for $4.5 \%$ of total disability-adjusted life years.(4)

The care of the post-cardiac arrest patient frequently involves a lengthy stay on the intensive care unit (ICU) with resource-intensive monitoring and high hospital costs.(5) In-hospital mortality is largely secondary to anoxic brain injury and withdrawal of life-sustaining therapy due to presumed poor prognosis.(6) It is an incredibly difficult time for family and friends of the patient involved, who are in need of objective information about the realistic chances of survival. An accurate prognostic assessment early in the post-OHCA pathway may be useful for medical teams to help guide families, and potentially to allow appropriate allocation of resources to those that are likely to benefit most, in an objective fashion.

Several scoring systems have been developed to determine the prognosis of patients presenting with OHCA. There are also many scores to assess neurological outcome, which can be used as an adjunct to those that predict mortality, however, those scores are also very heterogeneous and a neurological outcome that may be an acceptable to some clinicians or relatives, may not be acceptable to others. It was our aim to review scores assessing survival post-OHCA, as this outcome is not subjective or fluctuant and can often be assessed very early on following OHCA. 
These scores have varying complexity, and some have limited practical application, such that there is currently no recommended simple scoring system for routine clinical use. We have undertaken a systematic review of OHCA prognostic scoring systems developed to date, to evaluate the comparative usefulness of these scores for the prediction of survival following OHCA.

\section{Methods}

We performed a systematic review of available risk scores to predict outcome in patients presenting with OHCA. This is reported in accordance with the Preferred Reporting Items for Systematic Reviews and Meta-Analyses.(7) For each score, we examined the details of original development and any subsequent validation cohorts, comparing the scores in terms of ease of use, ability to predict survival and availability of independent external validation.

\section{Data Sources}

A systematic search of online databases Embase, MEDLINE and Cochrane Central Register of Controlled Trials was conducted from the earliest publication date available up until 20th November 2020 using the search strategy in Supplement 1. The reference lists of included studies identified in the search were screened for additional studies. After removal of duplicates, the titles and abstracts of the search results were evaluated for relevance by two reviewers (YG, KA). The full texts of the remaining results were independently assessed by both authors, for inclusion based on pre-determined criteria as per Study Selection, below. The final list of included studies was agreed by discussion between all authors, with full agreement required before inclusion. No disagreements required resolution by a third reviewer. 
Study Selection

The following inclusion criteria were applied: 1) studies involving individuals presenting with OHCA, 2) studies investigating survival outcomes of individuals with initial return of spontaneous circulation (ROSC), 3) studies investigating the use of any risk / predictive scoring system for predicting mortality outcomes following admission. The following studies were excluded: 1) only abstract available, 2) not reporting outcome of interest.

\section{Data Extraction}

Data were independently extracted from relevant published articles by two authors (YG, KA) which included number and type of patients included, risk score utilised, method of score calculation, score cut-off used, and predicted mortality outcome(s) reported, ranging from inhospital to 90-day mortality (including sensitivity, specificity, positive predictive value [PPV] and negative predictive value [NPV] as available).

\section{Funding and contribution}

No extramural funding was used to support this work. The authors are solely responsible for the design and conduct of this study, all study analyses and drafting and editing of the paper.

\section{Results}

The systematic search identified 1817 articles, from which 16 articles were included in this review (Figure 1). A total of 28 scoring systems were identified which assessed patients on admission, 11 of the scores included predicted mortality following OHCA, 14 predicted neurological outcome and 3 scores, not specific to the OHCA-population including the GRACE(8) (which was developed for use in mortality prediction in acute coronary 
syndrome), the APACHE II(9) and the SOFA(10) scores (used for mortality prediction in the ICU), which have been validated in an OHCA cohort (Supplement 2).

All the 11 scoring systems predicting mortality included in this review were developed specifically for this purpose(11-20) except the OHCA score(21), which was initially developed to assess neurological function on discharge but has been used in validation cohorts to assess mortality outcome.

Table 1 shows the 11 scores validated to predict survival after OHCA. The components, how it is calculated, outcome predicted and corresponding AUC for predicting outcome and the population used for development and validation are shown. The breakdown of different components and frequency of each as utilised in risk scores included in this review are shown in Supplement 3.

Summary of main scoring systems

Advanced Cardiac Life Support (ACLS) Score

The ACLS score was developed in 1981, using retrospective data from 611 patients with OHCA attributable to a cardiac aetiology.(11) The authors identified 4 variables predictive of in-hospital mortality, looking purely at arrest characteristics. The main advantages of the score are the ease-of-calculation, the clear indication of outcome specifying the percentage likelihood of survival and the components are part of the recommended Utstein-style reporting guidelines.(22) Disadvantages include development in an historical cohort treated with non-contemporary management, restriction to OHCA of cardiac aetiology, and poor predictive value (AUC 0.33(23) and $0.786(15)$ ) on external validation in undifferentiated, similarly sized cohorts (575 and 591 patients, respectively). 


\section{Graphic Model}

A second risk predictor model was developed using data from 1,667 patients with witnessed ventricular fibrillation arrest, of cardiac aetiology.(12) A regression model was used to determine the impact of a different set of arrest characteristics, namely time to cardiopulmonary resuscitation (CPR), defibrillation and delivery of advanced cardiac life support (ACLS) on survival. Similar to the ACLS score, this was developed in a noncontemporary cohort and required the recollection of time to events, subject to bias, which would have significant impact on the score and therefore reduce the usefulness and reliability. We were unable to identify any subsequent validation.

\section{Cardiac Arrest Score}

This score was derived from 127 patients with witnessed OHCA secondary to a cardiac cause.(13) This score uses a combination of arrest characteristics with initial blood pressure and neurological status. The score is simple to use, allocating a point to unfavourable characteristics i.e. long CPR time, low blood pressure and poor neurological status on assessment. However, the small development cohort and lack of external validation are major weaknesses.

\section{Decision Tree Model}

Data from 754 OHCA patients were used to develop a simple flowchart to predict a binary outcome - death or survival to discharge.(14) The model further differentiates depending on the Glasgow Coma Scale (GCS) post-ROSC. It has a high PPV, a measure of the accuracy of its prediction that patients with predicted mortality will die, of $99.4 \%$. However, the NPV, namely the accuracy its prediction of patients that will live, is only $12.5 \%$. The advantage of this model is that it is simple to use. The main shortcoming is that it only provides a binary 
outcome and does not provide clinicians with predicted probability of survival. Furthermore, the use of the GCS in this setting may not be reliable as emergency medical services may have administered neuromuscular blocking agents to facilitate advanced airway management prior to hospital admission.

\section{Out-of-Hospital Cardiac Arrest (OHCA) Score}

This score was developed in France comprising of several arrest characteristics and immediate laboratory parameters, to predict neurological outcome assessed using Cerebral Performance Categories of the Glasgow-Pittsburgh Outcome Categories.(21) The OHCA score is presented as a continuous scale and has been externally validated with good predictive value (AUC 0.74 for 30-day mortality(24) and 0.85 for in-hospital mortality(25)). The main limitation is that the calculation of the score is complicated and difficult to compute, and requires accurate measurement of timings during the arrest, which are frequently not available or subject to recall bias.

\section{Serbian Quality of Life short-term (SR-QOLs) Score}

This score was developed from a 2-year prospective multicentre study involving $591 \mathrm{OHCA}$ patients, from which 3 predictive scores for immediate, short-term and long-term prediction were derived.(15) Short-term survival, defined as survival to hospital discharge, has an AUC of 0.882 and the score is relatively easy to compute and requires minimal data, with the added advantage of having different scores depending on prediction required (immediate vs. shortterm vs. long-term). However, the score has not been validated externally. 


\section{Simple Prognostication Score}

This score was derived from 750 OHCA patients and includes 10 different parameters including arrest characteristics, patient clinical status on arrival, blood test results and the aetiology of arrest, with each variable weighted similarly.(16) The score was used to predict neurological status and survival rate 1-month post-arrest. A score of 6 or more has a sensitivity of $88.6 \%$ and specificity of $97.6 \%$ for 30 -day mortality. This score is simple to calculate, has high sensitivity and specificity and requires information that is readily available in most hospitals. The maximum score of 9 implies $100 \%$ mortality rate. However, no external validation has been performed.

\section{Prediction Tool}

This tool to predict 30-day mortality was developed using a developmental dataset of 1068 patients and further validated internally in 291 patients.(17) It is based on several arrest characteristics and the age of the patient, with different weighting assigned to the nominal scale of each variable, which are added to compute the final score. Although the AUC is 0.81 , there has been no external validation and the score is difficult to calculate due to the different cut-offs.

\section{NULL-PLEASE Score}

The NULL-PLEASE score is a futility score which assigns points to unfavourable arrest and

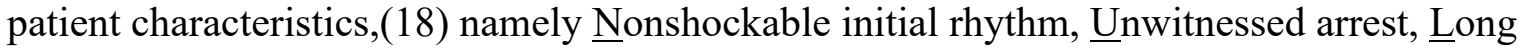
low-flow period, Long no-flow period, $\mathrm{pH}<7.2$, Lactate $>7.0 \mathrm{mmol} / \mathrm{L}$, End-stage renal

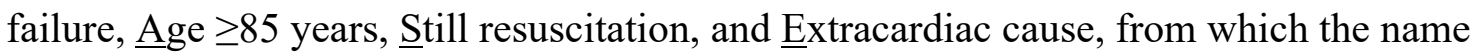
derives. 
This score was initially developed through a small retrospective cohort of 56 patients and a score of $>6$ was predictive of $100 \%$ in-hospital mortality. The score is easy to calculate as each of the initial arrest characteristics are assigned 2 points whilst the others are each assigned 1 point.

Recent independent external validation in a contemporary cohort(26) shows an AUC of 0.874 for predicting in-hospital mortality. A score of $\geq 3$ has a specificity of $93.4 \%$ but low sensitivity at $57.3 \%$. Similar to the development cohort(18), validation cohort showed a score of $>6$ was associated with $100 \%$ mortality. The NULLPLEASE score of $\geq 3$ has a high PPV of $90.6 \%$ and good NPV of $70.9 \%$. Another external validation was performed within a single-centre cohort,(27) since the outcome was not in-hospital mortality, it was not included.

\section{Polish Hypothermia Registry Risk Score (PHR-RS)}

The recently published PHR-RS,(19) was developed from the data of 376 patients who received TTM, to identify characteristics which were predictive of death; namely age and components of the mild therapeutic hypothermia score which mainly comprises of arrest characteristics. The score showed an AUC of 0.74 for predicting in-hospital death, but is complicated to calculate and has not been externally validated.

\section{Revised post-Cardiac Arrest Syndrome for Therapeutic hypothermia (rCAST) Score}

The rCAST score was developed from prospective, multicentre data on 460 patients in the Japanese Association for Acute Medicine Out-of-Hospital Cardiac Arrest registry.(20) Patients with traumatic OHCA, those not receiving TTM, and those under the age of 18 were excluded. The score incorporates only a single arrest characteristic, blood test results and the GCS, but the score is calculated based on cut-off values within each component and therefore 
requires reference to the score calculator to calculate. The score has an AUC of 0.832 at 30 days and 0.827 at 90 days and has not been externally validated. The exclusion of patients not receiving TTM introduces a selection bias in evaluating the usefulness of this score and therefore it may not be as useful in contemporary practice.

\section{Discussion}

A useful scoring system should provide high sensitivity (to predict patients with poor prognosis) and high specificity (to ensure all patients with potentially good outcomes are treated)(28,29). This systematic review has identified many risk scores from the current literature with variable predictive ability in this high-risk cohort of patients which reflects firstly, the emphasis on having an admission prognosis prediction and secondly, the difficulty in providing a "perfect" risk prediction tool. Furthermore, although these scores can be useful to provide initial prognostic information, further prognostic assessment at least 3 days postarrest remains the recommendation from international guidelines.

\section{Comparing risk scores}

When comparing the performance of the predictive scores, one needs to remember that these have often been developed and validated in differing cohorts of OHCA patients, including undifferentiated OHCA patients in some studies, different aetiologies of OHCA (mostly excluding traumatic OHCA), patients treated with TTM, and variable neurological status post-arrest. This has made comparing the various scores challenging, as the starting point is diverse. Furthermore, there is significant variation in the AUC reported for different scores between the development and validation cohorts, with variation from $0.33-0.88$ in predicting mortality. 
In trying to determine the optimal risk score to use to predict survival following OHCA, we have considered the ease of use, the size of the development cohorts, the predictive value and extent of independent validation. Scores developed in cohorts before the 1990s do not reflect contemporary treatments and also overall offer poorer predictive value (smaller AUC). Risk scores that offer good predictive ability, namely AUC $>0.8$, include the OHCA,(21) SRQOLs,(15) Prediction tool,(17) NULL-PLEASE (18) and rCAST(20) scores and these should be considered initially, based on the availability of clinical data required in the score. In situations where minimal data are available, use of the Prediction tool and SR-QOLs can be considered, as these only require arrest characteristics together with either the patient's age or pupil reactivity, respectively, although these scores have not been externally validated. When more information is available, including blood results on admission, the OHCA, NULLPLEASE and rCAST scores can be used and appear to perform similarly. However, the OHCA score requires the use of a calculator and similarly, the rCAST score requires reference to the score to identify the cut-off values of each component of the score, making both these unwieldy, when simpler scores which are easier to use, such as the NULLPLEASE score, provide similar predictive information (AUC 0.873 on external validation).

\section{Sensitivity/Specificity vs. Negative/Positive predictive value}

The true value of a predictive score can be viewed as that of a "screening" test for a condition, namely the likelihood that a score will reliably identify the outcome within an individual. This has been variably represented in the literature by the AUC, from which a cindex may be calculated, and the sensitivity and specificity of a particular test, and the PPV and NPV. Whilst most predictive risk scores provide AUC, a few provide sensitivity and specificity, and only two provide PPV and NPV (Table 1). 
It is important for clinicians who employ such a tool to recognise the distinction between sensitivity and specificity, which indicate the effectiveness of a test with respect to a trusted outcome, and PPV and NPV, where the latter indicate the effectiveness of a test for categorizing people as at risk or not at risk of having the outcome. More precisely, sensitivity and specificity indicate the concordance of a test with respect to a chosen outcome or condition, while PPV and NPV, respectively, indicate the likelihood that the test can successfully identify whether people do or do not have the outcome, based on their test results.(30)

The two contexts (i.e., the context that relates to sensitivity and specificity, versus the context that relates to the two predictive values) should not be confused with each other. Although extremely useful to assess the performance of a test, sensitivity and specificity should not be relied on when making decisions about individual people developing or having a particular condition or outcome. For the individual, use of PPVs and NPVs is more appropriate especially in a condition with high mortality,(31) and can be easily interpreted by family and next of kin, as the PPV for mortality indicates the probability that the individual will succumb to the illness. 
Score components with prognostic importance

The frequency with which different components are included in each risk score provides a rough indication of which score components have most prognostic significance (Supplemental Table 3). These include arrest-related characteristics such as initial rhythm (shockable versus non-shockable) and time to ROSC. Other patient-related factors such as age also frequently feature in different risk scores.

\section{Clinical use of a predictive risk score in OHCA}

Risk scores that predict risk or outcome are frequently used in everyday clinical practice, to guide prognosis and treatment, such as the $\mathrm{CHA}_{2} \mathrm{DS}_{2}$-Vasc score,(32) which is used to help clinicians risk-stratify patients with atrial fibrillation to identify the risk of stroke and systemic embolism, and then in turn, assess the potential benefit of antithrombotic therapy. Whilst this can be a very important decision, in the setting of OHCA, the situation is very different, in part because of the immediacy of the need to guide relatives; the risk of basing treatment wrongly on the predictive risk score which could then make the difference between life and death; and because the patient cannot input into the therapeutic decision making. It appears wholly unethical to make a decision to continue or withdraw life-saving treatment based on any risk score that does not have $100 \%$ predictive value. Risk scores should instead be used as an adjunct to help doctors provide objective, non-emotive and reproducible information to their next-of-kin on prognosis, as well as for medical teams as an addition to the clinical information available.

\section{Limitations}


Publication bias is one of the main limitations of this review as studies with smaller impact may not have been published. The varied presentation and heterogenicity of patients presenting with OHCA within each paper is not always available and may have an impact on the predictive value provided. There has been no formal head-to-head comparison of the various risk scores although this could be possible with pooling analysis using individual patient-level data. Outcome following OHCA may vary by different regions/centres and therefore the risk scores may not be universally applicable to different populations, healthcare providers or systems.

\section{Conclusion}

There are a number of risk scores available to determine the likelihood of survival following OHCA. The OHCA, NULL-PLEASE and rCAST scores appear most favourable and perform similarly in predicting survival, and of these, we recommend the NULL-PLEASE score as the one that is easiest to calculate and which has been externally validated. Risk scores should be used by medical professionals to provide objective, non-emotive and reproducible information to next-of-kin, but in isolation, should not form the basis for clinical decision making, and more definitive prognostic assessment should be performed at least 3 days postOHCA in line with guidelines. 


\section{Funding}

None.

\section{Patient and public involvement}

There was no patient or public involvement in this project.

\section{Ethics}

As this was a systematic review not involving direct patient care or data, ethical approval was not sought.

\section{Disclosures}

The authors have no disclosures to make in respect of this manuscript.

\section{Authors' contribution}

YXG: Conceptualization, Methodology, Data curation, Writing - original draft, Visualization KA: Methodology, Investigation

RK: Methodology, Investigation

TP: Conceptualization, Methodology, Validation

GYHL: Conceptualization, Methodology, Validation

DAG: Conceptualization, Methodology, Validation, Writing - Review and Editing, Visualisation, Supervision. 


\section{References}

1. Virani SS, Alonso A, Benjamin EJ, Bittencourt MS, Callaway CW, Carson AP, et al. Heart disease and stroke statistics_-2020 update: A report from the American Heart Association [Internet]. Vol. 141, Circulation. Lippincott Williams and Wilkins; 2020 [cited 2020 Nov 13]. p. E139-596. Available from: https://pubmed.ncbi.nlm.nih.gov/31992061/

2. Berdowski J, Berg RA, Tijssen JGP, Koster RW. Global incidences of out-of-hospital cardiac arrest and survival rates: Systematic review of 67 prospective studies. Resuscitation. 2010 Nov;81(11):1479-87.

3. Sasson C, Rogers MAM, Dahl J, Kellermann AL. Predictors of Survival From Out-ofHospital Cardiac Arrest. Circ Cardiovasc Qual Outcomes. 2010 Jan;3(1):63-81.

4. Coute RA, Nathanson BH, Panchal AR, Kurz MC, Haas NL, McNally B, et al. Disability-Adjusted Life Years Following Adult Out-of-Hospital Cardiac Arrest in the United States. Circ Cardiovasc Qual Outcomes. 2019 Mar;12(3):e004677.

5. Nolan JP, Soar J, Cariou A, Cronberg T, Moulaert VRM, Deakin CD, et al. European Resuscitation Council and European Society of Intensive Care Medicine Guidelines for Post-resuscitation Care 2015. Resuscitation. 2015;95:202-22.

6. Lemiale V, Dumas F, Mongardon N, Giovanetti O, Charpentier J, Chiche J-D, et al. Intensive care unit mortality after cardiac arrest: the relative contribution of shock and brain injury in a large cohort. Intensive Care Med. 2013 Nov;39(11):1972-80.

7. Moher D, Liberati A, Tetzlaff J, Altman DG, PRISMA Group. Preferred reporting items for systematic reviews and meta-analyses: the PRISMA Statement. Open Med. 2009;3(3):e123-30.

8. Fox KAA, Dabbous OH, Goldberg RJ, Pieper KS, Eagle KA, Van De Werf F, et al. Prediction of risk of death and myocardial infarction in the six months after 
presentation with acute coronary syndrome: Prospective multinational observational study (GRACE). Br Med J. 2006 Nov 25;333(7578):1091-4.

9. Knaus WA, Draper EA, Wagner DP, Zimmerman JE. APACHE II: a severity of disease classification system. Crit Care Med. 1985 Oct;13(10):818-29.

10. Vincent JL, Moreno R, Takala J, Willatts S, De Mendonça A, Bruining H, et al. The SOFA (Sepsis-related Organ Failure Assessment) score to describe organ dysfunction/failure. On behalf of the Working Group on Sepsis-Related Problems of the European Society of Intensive Care Medicine. Intensive Care Med. 1996 Jul;22(7):707-10.

11. Eisenberg M, Hallstrom A, Bergner L. The ACLS Score. JAMA. 1981 Jul 3;246(1):50.

12. Larsen MP, Eisenberg MS, Cummins RO, Hallstrom AP. Predicting survival from outof-hospital cardiac arrest: A graphic model. Ann Emerg Med. 1993;22(11):1652-8.

13. Thompson RJ, McCullough PA, Kahn JK, O’Neill WW. Prediction of death and neurologic outcome in the emergency department in out-of-hospital cardiac arrest survivors. Am J Cardiol. 1998 Jan 1;81(1):17-21.

14. Haukoos JS, Lewis RJ, Niemann JT. Prediction rules for estimating neurologic outcome following out-of-hospital cardiac arrest. In: Resuscitation. 2004. p. 145-55.

15. Sladjana A. A prediction survival model for out-of-hospital cardiopulmonary resuscitations. J Crit Care. 2011;26(2):223.e11-223.e18.

16. Ishikawa S, Niwano S, Imaki R, Takeuchi I, Irie W, Toyooka T, et al. Usefulness of a simple prognostication score in prediction of the prognoses of patients with out-ofhospital cardiac arrests. Int Heart J. 2013;54(6):362-70.

17. Aschauer S, Dorffner G, Sterz F, Erdogmus A, Laggner A. A prediction tool for initial out-of-hospital cardiac arrest survivors. Resuscitation. 2014 Sep;85(9):1225-31.

18. Ahmad R, Lumley S, Lau YC. NULL-PLEASE: A new 'Futility score' in the 
management of survivors of out-of-hospital cardiac arrest. Resuscitation. 2016 Sep 1;106:e83.

19. Kołtowski Ł, Średniawa B, Tycińska A, Czajkowska M, Niedziela M, Puchalski W, et al. Predicting survival in out-of-hospital cardiac arrest patients undergoing targeted temperature management: The Polish Hypothermia Registry Risk Score. Cardiol J. 2019 Apr 17;

20. Nishikimi M, Ogura T, Nishida K, Takahashi K, Nakamura M, Matsui S, et al. External validation of a risk classification at the emergency department of post-cardiac arrest syndrome patients undergoing targeted temperature management. Resuscitation. 2019 Jul 1;140:135-41.

21. Adrie C, Cariou A, Mourvillier B, Laurent I, Dabbane H, Hantala F, et al. Predicting survival with good neurological recovery at hospital admission after successful resuscitation of out-of-hospital cardiac arrest: the OHCA score. Eur Heart J. 2006 Nov $13 ; 27(23): 2840-5$.

22. Cummins RO, Chamberlain DA, Abramson NS, Allen M, Baskett PJ, Becker L, et al. Recommended guidelines for uniform reporting of data from out-of-hospital cardiac arrest: the Utstein Style. A statement for health professionals from a task force of the American Heart Association, the European Resuscitation Council, the Heart and Stroke. Circulation. 1991 Aug;84(2):960-75.

23. Haukoos JS, Lewis RJ, Stratton SJ, Niemann JT. Is the ACLS score a valid prediction rule for survival after cardiac arrest? Acad Emerg Med. 2003 Jun 1;10(6):621-6.

24. Choi JY, Jang JH, Lim YS, Jang JY, Lee G, Yang HJ, et al. Performance on the APACHE II, SAPS II, SOFA and the OHCA score of post-cardiac arrest patients treated with therapeutic hypothermia. PLoS One. 2018;13(5):e0196197.

25. Hunziker S, Bivens MJ, Cocchi MN, Miller J, Salciccioli J, Howell MD, et al. 
International validation of the out-of-hospital cardiac arrest score in the United States. Crit Care Med. 2011;39(7):1670-4.

26. Gue YX, Sayers M, Whitby B, Kanji R, Adatia K, Smith R, et al. Usefulness of the NULL-PLEASE Score to predict survival in out-of-hospital cardiac arrest. Am J Med. 2020;

27. Potpara TS, Mihajlovic M, Stankovic S, Jozic T, Jozic I, Asanin MR, et al. External Validation of the Simple NULL-PLEASE Clinical Score in Predicting Outcome of Out-of-Hospital Cardiac Arrest. Am J Med. 2017 Dec;130(12):1464.e13-1464.e21.

28. Sunde K, Kramer-Johansen J, Pytte M, Steen PA. Predicting survival with good neurologic recovery at hospital admission after successful resuscitation of out-ofhospital cardiac arrest: the OHCA score. Eur Heart J. 2007 Feb 28;28(6):773-773.

29. Gold B, Puertas L, Davis SP, Metzger A, Yannopoulos D, Oakes DA, et al. Awakening after cardiac arrest and post resuscitation hypothermia: Are we pulling the plug too early? Resuscitation. 2014 Feb;85(2):211-4.

30. Trevethan R. Sensitivity, Specificity, and Predictive Values: Foundations, Pliabilities, and Pitfalls in Research and Practice. Front Public Heal. 2017 Nov 20;5(307).

31. Akobeng AK. Understanding diagnostic tests 1: Sensitivity, specificity and predictive values. Vol. 96, Acta Paediatrica, International Journal of Paediatrics. 2007. p. 338-41.

32. Lip GYH, Nieuwlaat R, Pisters R, Lane DA, Crijns HJGM, Andresen D, et al. Refining clinical risk stratification for predicting stroke and thromboembolism in atrial fibrillation using a novel risk factor-based approach: The Euro Heart Survey on atrial fibrillation. Chest. 2010 Feb 1;137(2):263-72. 